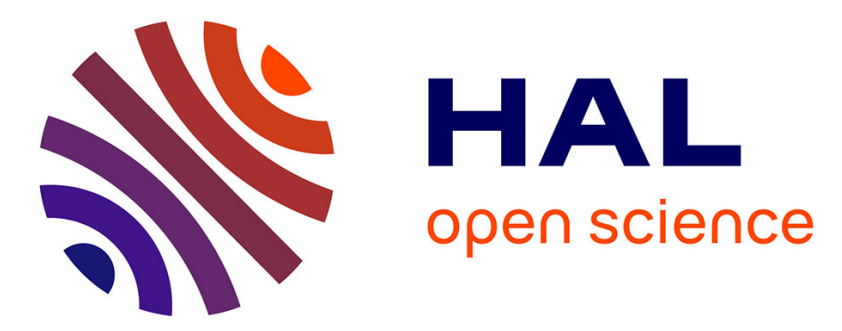

\title{
RFC7182: Integrity Check Value and Timestamp TLV Definitions for Mobile Ad Hoc Networks (MANETs)
}

Thomas Heide Clausen, Ulrich Herberg, Christopher Chris Dearlove

\section{To cite this version:}

Thomas Heide Clausen, Ulrich Herberg, Christopher Chris Dearlove. RFC7182: Integrity Check Value and Timestamp TLV Definitions for Mobile Ad Hoc Networks (MANETs). [Technical Report] RFC7182, The Internet Engineering Task Force. 2014. hal-03172446

HAL Id: hal-03172446

https://hal-polytechnique.archives-ouvertes.fr/hal-03172446

Submitted on 17 Mar 2021

HAL is a multi-disciplinary open access archive for the deposit and dissemination of scientific research documents, whether they are published or not. The documents may come from teaching and research institutions in France or abroad, or from public or private research centers.
L'archive ouverte pluridisciplinaire HAL, est destinée au dépôt et à la diffusion de documents scientifiques de niveau recherche, publiés ou non, émanant des établissements d'enseignement et de recherche français ou étrangers, des laboratoires publics ou privés. 


\section{Integrity Check Value and Timestamp TLV Definitions for Mobile Ad Hoc Networks (MANETs)}

Abstract

This document revises, extends, and replaces RFC 6622. It describes general and flexible TLVs for representing cryptographic Integrity Check Values (ICVs) and timestamps, using the generalized Mobile Ad Hoc Network (MANET) packet/message format defined in RFC 5444. It defines two Packet TLVs, two Message TLVs, and two Address Block TLVs for affixing ICVs and timestamps to a packet, a message, and one or more addresses, respectively.

Status of This Memo

This is an Internet Standards Track document.

This document is a product of the Internet Engineering Task Force (IETF). It represents the consensus of the IETF community. It has received public review and has been approved for publication by the Internet Engineering Steering Group (IESG). Further information on Internet Standards is available in section 2 of RFC 5741.

Information about the current status of this document, any errata, and how to provide feedback on it may be obtained at http://www.rfc-editor.org/info/rfc7182. 


\section{Copyright Notice}

Copyright (c) 2014 IETF Trust and the persons identified as the document authors. All rights reserved.

This document is subject to BCP 78 and the IETF Trust's Legal Provisions Relating to IETF Documents

(http://trustee.ietf.org/license-info) in effect on the date of publication of this document. Please review these documents carefully, as they describe your rights and restrictions with respect to this document. Code Components extracted from this document must include Simplified BSD License text as described in section 4.e of the Trust Legal Provisions and are provided without warranty as described in the Simplified BSD License.

\section{Table of Contents}

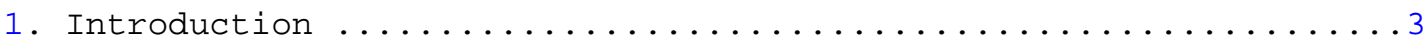

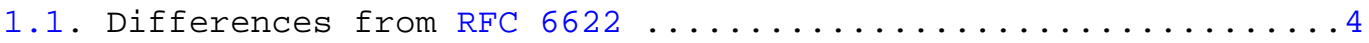

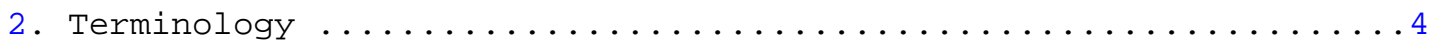

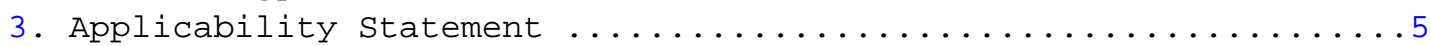

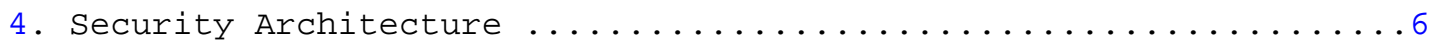

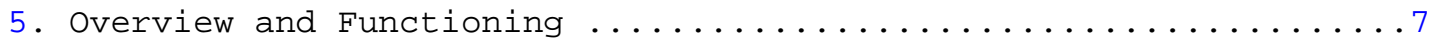

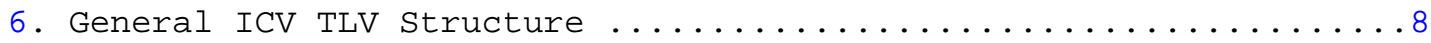

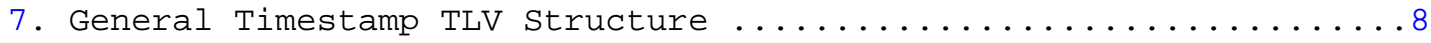

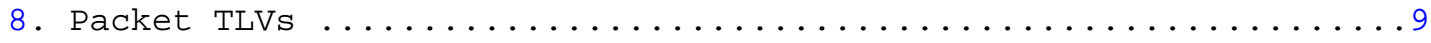

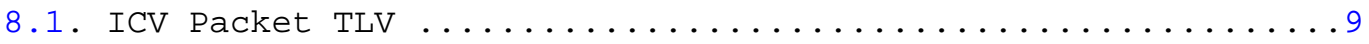

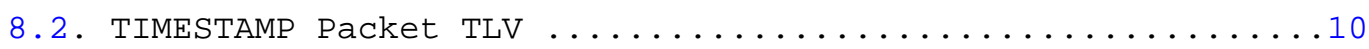

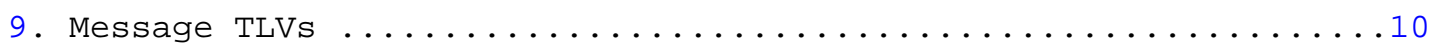

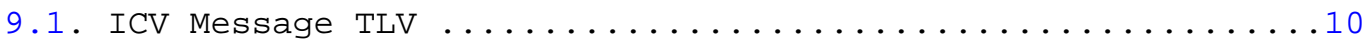

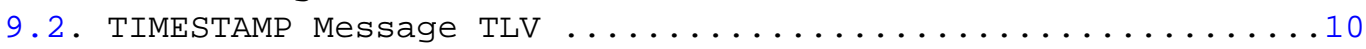

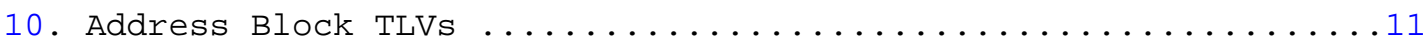

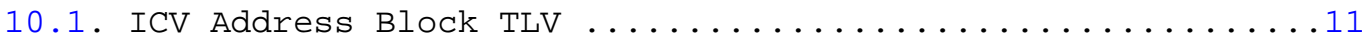

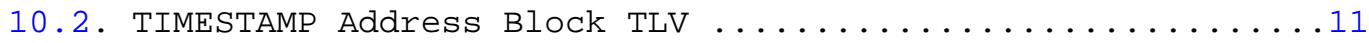

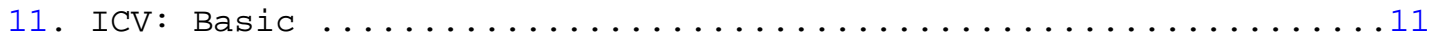

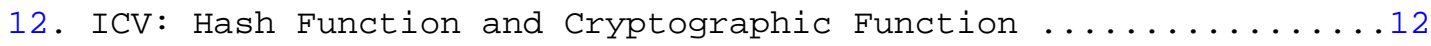

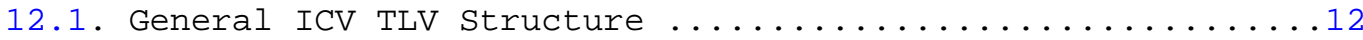

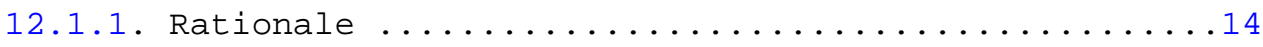

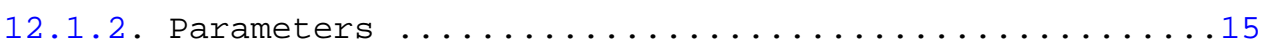

12.2. Considerations for Calculating the ICV ............. 15

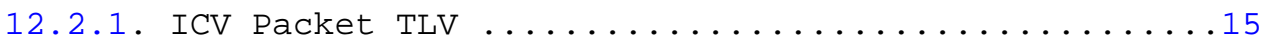

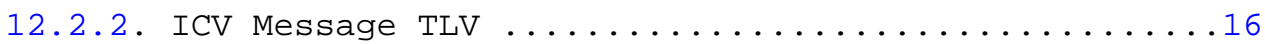

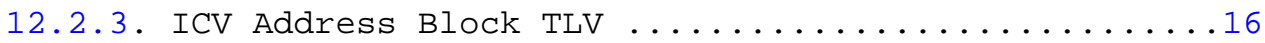

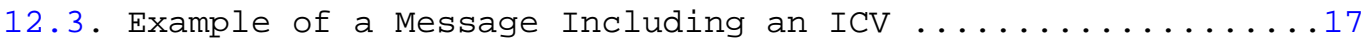

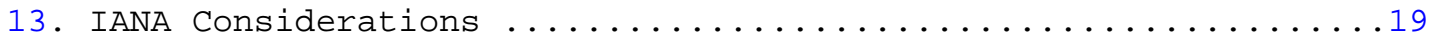

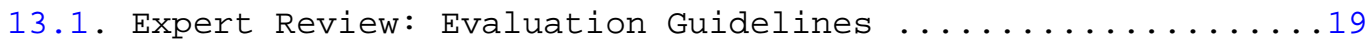

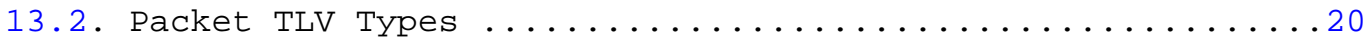

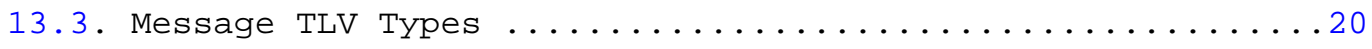




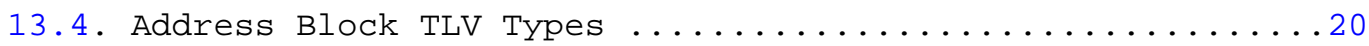

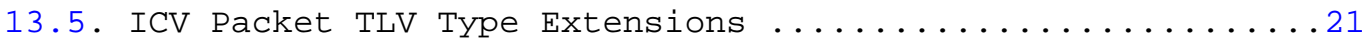

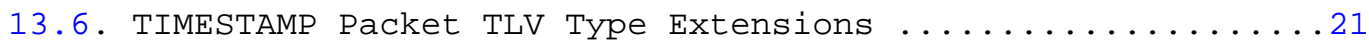

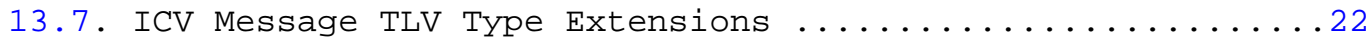

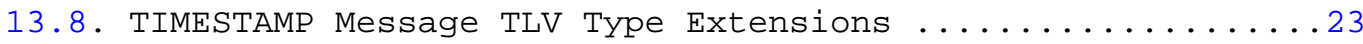

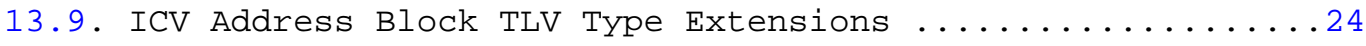

13.10. TIMESTAMP Address Block TLV Type Extensions .........25

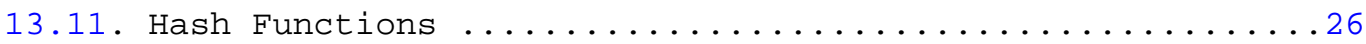

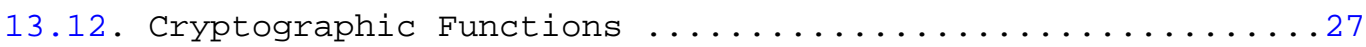

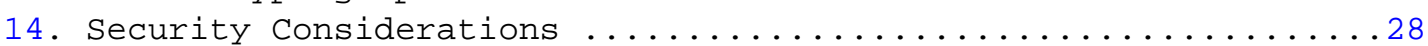

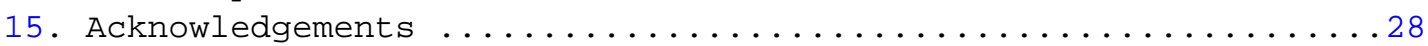

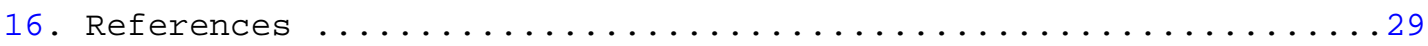

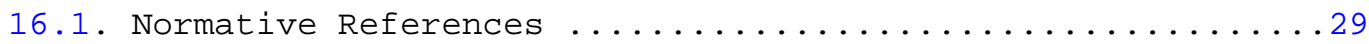

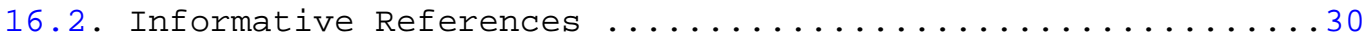

\section{Introduction}

This document specifies a syntactical representation of securityrelated information for use with [RFC5444] addresses, messages, and packets. It also specifies IANA registrations of TLV types and type extension registries for these TLV types. This specification does not represent a stand-alone protocol, but it is intended for use by MANET routing protocols or security extensions thereof.

Specifically, this document, which revises, extends, and replaces [RFC6622], specifies:

- Two kinds of TLV: one for carrying Integrity Check Values (ICVs) and one for timestamps in packets, messages, and Address Blocks as defined by [RFC5444].

- A generic framework for use of these TLVs, accounting for specific features of Packet, Message, and Address Block TLVs.

- IANA registrations for TLVs, and registries for TLV type extensions, replacing those from [RFC6622].

This document specifies IANA registries for recording code points for ICV TLVs and TIMESTAMP TLVs, as well as timestamps, hash functions, and cryptographic functions.

Moreover, in Section 12, this document defines the following:

- A method for generating ICVs using a combination of a cryptographic function and a hash function and for including such ICVs in the value field of a TLV. 


\subsection{Differences from RFC 6622}

This document obsoletes [RFC6622], replacing that document as the specification of two TLV types, TIMESTAMP and ICV, for packets, messages and Address Blocks. For the ICV type, this document specifies a new type extension, 2 (see Section 12), in addition to including the original type extensions (0 and 1) from [RFC6622].

The TLV value of an ICV TLV with type extension $=2$ has the same internal structure as an ICV TLV with type extension $=1$ but is calculated also over the source address of the IP datagram carrying the packet, message, or Address Block. The rationale for adding this type extension is that some MANET protocols, such as [RFC6130], use the IP source address of the IP datagram carrying the packet, message, or Address Block, e.g., to identify links with neighbor routers. If this address is not otherwise contained in the packet, message, or Address Block payload (which is permitted, e.g., in [RFC6130]), then the address is not protected against tampering.

This document also incorporates a number of editorial improvements over [RFC6622]. In particular, it makes it clear that an ICV TLV may be used to carry a truncated ICV and that a single or multivalue TIMESTAMP or ICV Address Block TLV may cover more than one address. Moreover, to be consistent with the terminology in [RFC5444], the name of the TLVs specified in this document have changed from "Packet ICV TLV" to "ICV Packet TLV" and from "Packet TIMESTAMP TLV" to "TIMESTAMP Packet TLV" (and similar for Message and Address Block TLVS).

A normative requirement in section 9.2 has changed from SHOULD to MUST in the following sentence:

If a message contains one or more TIMESTAMP TLVs and one or more ICV TLVs, then the TIMESTAMP TLVs (as well as any other Message TLVS) MUST be added to the message before the ICV TLVs...

2. Terminology

The key words "MUST", "MUST NOT", "REQUIRED", "SHALL", "SHALL NOT", "SHOULD", "SHOULD NOT", "RECOMMENDED", "NOT RECOMMENDED", "MAY", and "OPTIONAL" in this document are to be interpreted as described in [RFC2119]. 
This document uses the terminology and notation defined in [RFC5444]. In particular, the following TLV fields and notation from [RFC5444] are used in this specification:

<msg-hop-limit> is the hop limit of a message, as specified in Section 5.2 of [RFC5444].

<msg-hop-count> is the hop count of a message, as specified in Section 5.2 of [RFC5444].

<length> is the length of the value field in a TLV in octets, as specified in section 5.4.1 of [RFC5444].

single-length is the length of a single value in the value field in a TLV in octets, as specified in section 5.4.1 of [RFC5444]. (It is equal to <length> except in a multivalue Address Block TLV.)

In addition to using the regular expressions defined in section 2.1.1 of [RFC5444], this document defines the following:

+ - One or more occurrences of the preceding element or group.

\section{Applicability statement}

MANET routing protocols using the format defined in [RFC5444] are accorded the ability to carry additional information in control messages and packets through the inclusion of TLVs. Information so included MAY be used by a MANET routing protocol, or by an extension of a MANET routing protocol, according to its specification.

This document specifies how to include an ICV for a packet, a message, and addresses in an Address Block within a message, using such TLVs. This document also specifies how to treat an empty Packet TLV Block, and "mutable" fields, specifically the <msg-hop-count> and <msg-hop-limit> fields, if present in the Message Header when calculating ICVs, such that the resulting ICV can be correctly verified by any recipient.

This document describes a generic framework for creating ICVs, and how to include these ICVs in TLVs. In Section 12, an example method for calculating such ICVs is given, using a cryptographic function and a hash function, for which two TLV type extensions are allocated.

This document does not specify a protocol. Protocol specifications that make use of the framework, specified in this document, will reference this document in a normative way, and they may require the implementation of some or all of the algorithms described in this document. As this document does not specify a protocol itself, key 
management and key exchange mechanisms are out of scope and may be specified in the protocol or protocol extension using this specification.

\section{Security Architecture}

MANET routing protocol specifications may have a clause allowing a control message to be rejected as "badly formed" or "insecure" prior to the message being processed or forwarded. In particular, MANET routing protocols such as the Neighborhood Discovery Protocol (NHDP) [RFC6130] and the Optimized Link State Routing Protocol version 2 [RFC7181] recognize external reasons (such as failure to verify an ICV) for rejecting a message that would be considered "invalid for processing".

This architecture is a result of the observation that with respect to security in MANETs, "one size rarely fits all" and that MANET routing protocol deployment domains have varying security requirements ranging from "unbreakable" to "virtually none". The virtue of this approach is that MANET routing protocol specifications (and implementations) can remain "generic", with extensions providing proper security mechanisms specific to a deployment domain.

The MANET routing protocol "security architecture", in which this specification situates itself, can therefore be summarized as follows:

- MANET routing protocol specifications, each with a clause allowing an extension to reject a message (prior to processing/forwarding) as "badly formed" or "insecure".

- MANET routing protocol security extensions, each rejecting messages as "badly formed" or "insecure", as appropriate for a given security requirement specific to a deployment domain.

- Code points and an exchange format for information, necessary for specification of such MANET routing protocol security extensions.

This document addresses the last of the points above, by specifying a common exchange format for cryptographic ICVs and timestamps, making reservations from within the Packet TLV, Message TLV, and Address Block TLV registries of [RFC5444], to be used by (and shared among) MANET routing protocol security extensions.

For the specific decomposition of an ICV using a cryptographic function and a hash function (specified in section 12), this document specifies two IANA registries (see section 13) for code points for hash functions and cryptographic functions. 
With respect to $[\mathrm{RFC} 5444]$, this document is:

- Intended to be used in the non-normative, but intended, mode of use described in Appendix B of [RFC5444].

- A specific example of the Security Considerations section of [RFC5444] (the authentication part).

5. Overview and Functioning

This document specifies a syntactical representation of securityrelated information for use with [RFC5444] addresses, messages, and packets, and also specifies IANA registrations (see section 13) of TLV types and type extension registries for these TLV types.

Moreover, this document provides guidelines for how MANET routing protocols, and MANET routing protocol extensions using this specification, should treat ICV and Timestamp TLVs, and mutable fields in messages. This specification does not represent a standalone protocol. MANET routing protocols, and MANET routing protocol extensions using this specification, MUST provide instructions as to how to handle packets, messages, and addresses with security information, associated as specified in this document.

This document specifies TLV type assignments (see Section 13) from the registries defined for Packet, Message, and Address Block TLVs in [RFC5444]. When a TLV type is assigned from one of these registries, a registry for type extensions for that TLV type is created by IANA. This document specifies these type extension registries, in order to specify internal structure (and accompanying processing) of the <value> field of a TLV.

For example, and as specified in this document, an ICV TLV with type extension $=0$ specifies that the $\langle$ value $\rangle$ field has no predefined internal structure, but is simply a sequence of octets. An ICV TLV with type extension $=1$ specifies that the <value $>$ field has a predefined internal structure and defines its interpretation. An ICV TLV with type extension $=2$ (added in this document) is the same as an ICV TLV with type extension $=1$, except that the integrity protection also covers the source address of the IP datagram carrying the packet, message, or Address Block.

Specifically, with type extension $=1$ or type extension $=2$, the <value> field contains the result of combining a cryptographic function and a hash function, calculated over the contents of the packet, message, or Address Block. The <value> field contains subfields indicating which hash function and cryptographic function have been used, as specified in section 12 . 
Other documents can request assignments for other type extensions; if they do so, they MUST specify their internal structure (if any) and interpretation.

6. General ICV TLV structure

The value of the ICV TLV is:

$\langle$ value $>:=\langle$ ICV-value $>+$

where:

<ICV-value> is a field, of length <length> octets (except in a multivalue Address Block TLV, where each <ICV-value> is of length single-length octets) that contains the information to be interpreted by the ICV verification process, as specified by the type extension.

Note that this does not specify how to calculate the <ICV-value $>$ nor the internal structure thereof, if any; such information MUST be specified by the type extension for the ICV TLV type; see Section 13. This document specifies three such type extensions: one for ICVs without predefined structures and two for ICVs constructed combining a cryptographic function and a hash function.

7. General Timestamp TLV Structure

The value of the Timestamp TLV is:

$\langle$ value $\rangle:=<$ time-value $>+$

where:

<time-value> is a field, of length <length> octets (except in a multivalue Address Block TLV, where each <time-value> is of length single-length octets) that contains the timestamp.

Note that this does not specify how to calculate the <time-value> nor the internal structure thereof, if any; such information MUST be specified by the type extension for the TIMESTAMP TLV type; see Section 13.

A timestamp is essentially "freshness information". As such, its setting and interpretation are to be determined by the MANET routing protocol, or MANET routing protocol extension, that uses the timestamp and can, for example, correspond to a POSIX timestamp, GPS timestamp, or a simple sequence number. Note that ensuring time synchronization in a MANET may be difficult because of the 
decentralized architecture as well as highly dynamic topology due to mobility or other factors. It is out of scope for this document to specify a time synchronization mechanism.

8. Packet TLVs

Two Packet TLVs are defined: one for including the cryptographic ICV of a packet and one for including the timestamp indicating the time at which the cryptographic ICV was calculated.

8.1. ICV Packet TLV

An ICV Packet TLV is an example of an ICV TLV as described in Section 6. When determining the <ICV-value> for a packet, and adding an ICV Packet TLV to a packet, the following considerations MUST be applied:

- Because packets as defined in [RFC5444] are never forwarded by routers, no special considerations are required regarding mutable fields (i.e., <msg-hop-count> and <msg-hop-limit>), if present within any messages in the packet, when calculating the ICV.

- Any ICV Packet TLVs already present in the Packet TLV Block MUST be removed before calculating the ICV, and the Packet TLV Block size MUST be recalculated accordingly.

- If the Packet TLV Block now contains no Packet TLVs, the Packet TLV Block MUST be removed, and the phastlv bit in the <pkt-flags> field in the Packet Header MUST be cleared ('0').

- Any removed ICV Packet TLVs MUST be restored after having calculated the ICV, and the Packet TLV Block size MUST be recalculated accordingly.

- When any removed ICV Packet TLVs, and the newly calculated ICV Packet TLV, are added to the packet, if there is no Packet TLV Block, then one MUST be added, including setting (' 1 ') the phastlv bit in the <pkt-flags field in the Packet Header.

The rationale for removing any ICV Packet TLVs already present prior to calculating the ICV is that several ICV TLVs may be added to the same packet, e.g., using different ICV cryptographic and/or hash functions. The rationale for removing an empty Packet TLV Block is because the receiver of the packet cannot tell the difference between what was an absent Packet TLV Block, and what was an empty Packet TLV Block when removing and verifying the ICV Packet TLV if no other Packet TLVs are present. 


\subsection{TIMESTAMP Packet TLV}

A TIMESTAMP Packet TLV is an example of a Timestamp TLV as described in section 7. If a packet contains one or more TIMESTAMP TLVs and one or more ICV TLVs, then the TIMESTAMP TLVs (as well as any other Packet TLVS) MUST be added to the packet before the ICV TLVs, in order to include the timestamps and other TLVs in the calculation of the ICVS.

9. Message TLVs

Two Message TLVs are defined: one for including the cryptographic ICV of a message and one for including the timestamp indicating the time at which the cryptographic ICV was calculated.

\subsection{ICV Message TLV}

An ICV Message TLV is an example of an ICV TLV as described in Section 6. When determining the <ICV-value> for a message, the following considerations MUST be applied:

o The fields <msg-hop-limit> and <msg-hop-count>, if present in the Message Header, MUST both be assumed to have the value 0 (zero) when calculating the ICV.

- Any ICV Message TLVs already present in the Message TLV Block MUST be removed before calculating the ICV, and the message size as well as the Message TLV Block size MUST be recalculated accordingly. Also, all relevant TLVs other than ICV TLVs MUST be added prior to ICV value calculation.

- Any removed ICV Message TLVs MUST be restored after having calculated the ICV, and the message size as well as the Message TLV Block size MUST be recalculated accordingly.

The rationale for removing any ICV Message TLVs already present prior to calculating the ICV is that several ICV TLVs may be added to the same message, e.g., using different ICV cryptographic and/or hash functions.

\subsection{TIMESTAMP Message TLV}

A TIMESTAMP Message TLV is an example of a Timestamp TLV as described in section 7. If a message contains one or more TIMESTAMP TLVs and one or more ICV TLVs, then the TIMESTAMP TLVs (as well as any other Message TLVS) MUST be added to the message before the ICV TLVs, in order to include the timestamps and other Message TLVs in the calculation of the ICV. 
10. Address Block TLVs

Two Address Block TLVs are defined: one for associating a cryptographic ICV to one or more addresses and their associated information and one for including the timestamp indicating the time at which the cryptographic ICV was calculated.

\subsection{ICV Address Block TLV}

An ICV Address Block TLV is an example of an ICV TLV as described in Section 6. The ICV is calculated over one or more addresses, concatenated with any other values -- for example, other Address Block TLV <value> fields -- associated with those addresses. A MANET routing protocol, or MANET routing protocol extension, using ICV Address Block TLVs MUST specify how to include any such concatenated attributes of the addresses in the calculation and verification processes for the ICV. When determining an <ICV-value> for one or more addresses, the following consideration MUST be applied:

- If other TLV values are concatenated with the addresses for calculating the ICV, the corresponding TLVs MUST NOT be ICV Address Block TLVs already associated with any of the addresses.

The rationale for not concatenating the addresses with any ICV TLV values already associated with the addresses when calculating the ICV is that several ICVs may be added to the same address or addresses, e.g., using different ICV cryptographic and/or hash functions, and the order of addition is not known to the recipient.

\subsection{TIMESTAMP Address Block TLV}

A TIMESTAMP Address Block TLV is an example of a Timestamp TLV as described in section 7. If one or more TIMESTAMP TLVs and one or more ICV TLVs are associated with an address, the relevant TIMESTAMP TLV <time-value>(s) MUST be included before calculating the value of the ICV to be contained in the ICV TLV value (i.e., concatenated with the associated addresses and any other values as described in Section 10.1).

\section{ICV: Basic}

The basic ICV, represented by way of an ICV TLV with type extension $=0$, has as TLV value a simple bit-field without specified structure (i.e, without explicitly included hash function, crypto function, key ID or other parameters). Moreover, it is not specified how to calculate the <ICV-value>. It is assumed that the mechanism specifying how ICVs are calculated and verified, as well as which parameters (if any) need to be exchanged prior to using the TLV with 
type extension $=0$, is established outside of this specification, e.g., by administrative configuration or external out-of-band signaling.

The <ICV-value>, when using type extension $=0$, is:

$$
\langle\text { ICV-value } \quad:=\langle\text { ICV-data }>
$$

where:

<ICV-data> is a field, of length <length> octets (or single-length octets in a multivalue Address Block TLV) that contains the cryptographic ICV.

12. ICV: Hash Function and Cryptographic Function

One common way of calculating an ICV is combining a cryptographic function and a hash function applied to the content. This decomposition is specified in this section, using either type extension $=1$ or type extension $=2$, in the ICV TLVs.

12.1. General ICV TLV Structure

The following data structure allows representation of a cryptographic ICV, including specification of the appropriate hash function and cryptographic function used for calculating the ICV:

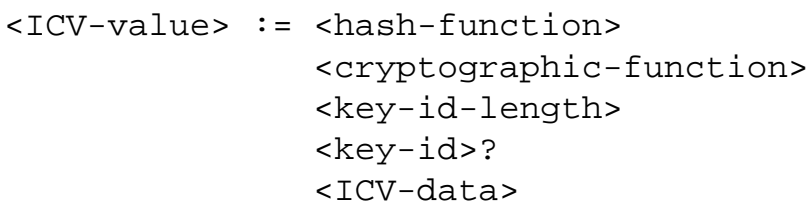

where:

<hash-function> is a one-octet unsigned integer field specifying the hash function.

<cryptographic-function> is a one-octet unsigned integer field specifying the cryptographic function.

<key-id-length> is a one-octet unsigned integer field specifying the length of the <key-id> field as a number of octets. The value zero $(0 \times 00)$ is reserved for using a single pre-installed, shared key . 
<key-id> is a field specifying the key identifier of the key that was used to calculate the ICV of the message, which allows unique identification of different keys with the same originator. It is the responsibility of each key originator to make sure that actively used keys that it issues have distinct key identifiers. If <key-id-length> equals zero (0x00), the <key-id> field is not contained in the TLV, and a single pre-installed, shared key is used.

<ICV-data> is a field with length <length> - 3 - <key-id-length> octets (except in a multivalue Address Block TLV, in which it is single-length - $3-<$ key-id-length> octets) and that contains the cryptographic ICV.

The version of this TLV, specified in this section, assumes that, unless otherwise specified, calculating the ICV can be decomposed into:

ICV-value = cryptographic-function(hash-function(content))

In some cases, a different combination of cryptographic function and hash function may be specified. This is the case for the Hashed Message Authentication Code (HMAC) function, which is specified as defined in Section 13.12, using the hash function twice. Using cryptographic-function "none" is provided for symmetry and possible future use, but it SHOULD NOT be used with any currently specified hash function.

The difference between the two type extensions is that in addition to the information covered by the ICV using type extension $=1$ (which is detailed in the following sections), the ICV using type extension $=2$ also MUST cover the source address of the IP datagram carrying the corresponding packet, message, or Address Block.

The <ICV-data> field MAY be truncated after being calculated, this is indicated by its length, calculated as described above. The truncation MUST be as specified for the relevant cryptographic function (and, if appropriate, hash function).

- When using truncation, the guidelines for minimal ICV length set out in [NIST-SP-800-107] MUST be followed. In particular the <ICV-data> field when using HMAC MUST NOT be truncated below 4 octets.

- The truncated ICV length MUST be so large that the probability of success of a dictionary attack is acceptably small. Such a success will arise if the ICV of a spoofed packet or message is verified. The probability of success is a function of (a) how 
many routers can be attacked, (b) how fast a router can receive packets or messages and attempt to verify their ICV, (c) the truncated ICV length, and (d) the lifetime of the network. If the truncated ICV length in bits is $\mathrm{L}$, then $2^{\wedge} \mathrm{L}$ packets or messages are required to attack with certainty of success. With a verification rate of $\mathrm{R}$ packets/messages per second, applied to $\mathrm{N}$ routers over an available time of $\mathrm{T}$, the probability of success is given by $\mathrm{NRT} / 2^{\wedge} \mathrm{L}$. If this is not to exceed a probability of $\mathrm{P}$, then $\mathrm{L}>\log 2(\mathrm{NRT} / \mathrm{P})$. For example, if $\mathrm{N}$ is $32, \mathrm{R}$ is $1000, \mathrm{~T}$ is 86400 ( $I$ day) and $P$ is $10^{\wedge}-6$, then $L$ must be at least 52 bits.

Some of the cryptographic and hash functions listed in Section 13 require the length of the content to be digitally signed to be a multiple of a certain number of octets. As a consequence, they specify padding mechanisms, e.g., AES-CMAC [RFC4493] specifies a padding mechanism for message lengths that are not equal to a multiple of 16 octets. Implementations of the framework in this document MUST support appropriate padding mechanisms, as specified in the cryptographic or hash function specifications.

The hash function and the cryptographic function correspond to the entries in two IANA registries, which are described in section 13.

\subsubsection{Rationale}

The rationale for separating the hash function and the cryptographic function into two octets instead of having all combinations in a single octet -- possibly as a TLV type extension -- is that adding further hash functions or cryptographic functions in the future may lead to a non-contiguous number space as well as a smaller overall space.

The rationale for not including a field that lists parameters of the cryptographic ICV in the TLV is that, before being able to validate a cryptographic ICV, routers have to exchange or acquire keys. Any additional parameters can be provided together with the keys in that bootstrap process. Therefore, it is not necessary, and would even entail an extra overhead, to transmit the parameters within every message.

The rationale for the addition of type extension $=2$ is that the source address is used in some cases, such as when processing HELLO messages in [RFC6130]. This is applicable only to packets (which only ever travel one hop) and messages (and their Address Blocks) that only travel one hop. It is not applicable to messages that may be forwarded more than one hop, such as Topology Control (TC) messages in [RFC7181]. 


\subsubsection{Parameters}

As described in section 12.1.1, parameters are selected administratively on each router before using this framework in a MANET, in addition to exchanging the keys between MANET routers. This was a design decision in [RFC6622] and is kept in this specification for reasons of backwards compatibility.

The following parameters are RECOMMENDED and SHOULD be those chosen administratively, unless there are good reasons otherwise:

- For crypto function RSA:

* Signature scheme: RSASSA-PSS with the default parameters: rSASSA-PSS-Default-Identifier (as defined in [RFC3447])

* Common exponent: 65537

- For crypto function ECDSA:

* Curve name: exchanged as part of key distribution

* Hash function: The hash function MUST be pinned to the curve, i.e., use SHA-256 for the p-256 curve, SHA-384 for p-384, etc.

- For crypto function AES:

* Authentication algorithm: Cipher-Based Message Authentication Code (CMAC) (as defined in [RFC4493])

* Hash function: None

12.2. Considerations for Calculating the ICV

The considerations listed in the following subsections MUST be applied when calculating the ICV for Packet, Message, and Address Block TLVs, respectively.

\subsubsection{ICV Packet TLV}

When determining the <ICV-data> for a packet, with type extension $=1$ :

- The ICV is calculated over the fields <hash-function>, <cryptographic-function>, <key-id-length>, and -- if present -$<$ key-id> (in that order), followed by the entire packet, including 
the Packet Header, including all Packet TLVs (other than ICV Packet TLVs), and all included messages. The considerations of Section 8.1 MUST be applied.

When determining the <ICV-data> for a packet, with type extension $=2$ :

- The same procedure as for type extension $=1$ is used, except that the data used consists of a representation of the source address of the IP datagram carrying the packet, followed by the remaining data (as for type extension $=1$ ). The representation of the source address consists of a single octet containing the address length, in octets, followed by that many octets containing the address in network byte order.

\subsubsection{ICV Message TLV}

When determining the <ICV-data> for a message, with type extension $=1$ :

- The ICV is calculated over the fields <hash-function>, <cryptographic-function>, <key-id-length>, and -- if present -$<$ key-id> (in that order), followed by the entire message. The considerations in Section 9.1 MUST be applied.

When determining the <ICV-data> for a message, with type extension $=2$ :

- The same procedure as for type extension $=1$ is used, except that the data used consists of a representation of the source address of the IP datagram carrying the message, followed by the remaining data (as for type extension $=1$ ). The representation of the source address consists of a single octet containing the address length, in octets, followed by that many octets containing the address in network byte order.

\subsubsection{ICV Address Block TLV}

When determining the <ICV-data> for one or more addresses, with type extension $=1$ :

- The ICV is calculated over the fields <hash-function>, <cryptographic-function>, <key-id-length>, and -- if present -<key-id> (in that order), followed by the addresses, and followed by any other values -- for example, other Address Block TLV <value>s that are associated with those addresses. A MANET routing protocol, or MANET routing protocol extension, using ICV Address Block TLVs MUST specify how to include any such 
concatenated attribute of the addresses in the verification process of the ICV. The consideration in section 10.1 MUST be applied.

When determining the <ICV-data> for one or more addresses, with type extension $=2$ :

- The same procedure as for type extension $=1$ is used, except that the data used consists of a representation of the source address of the IP datagram carrying the Address Block, followed by the remaining data (as for type extension = 1). The representation of the source address consists of a single octet containing the address length, in octets, followed by that many octets containing the address in network byte order.

12.3. Example of a Message Including an ICV

The sample message depicted in Figure 1 is derived from Appendix $E$ of [RFC5444]. The message contains an ICV Message TLV, with the value representing an ICV that is 16 octets long and a key identifier that is 4 octets long. The type extension of the Message TLV is 1 , for the specific decomposition of an ICV using a cryptographic function and a hash function, as specified in section 12. 
Figure 1: Example Message with ICV 
MF: $\quad$ Message Flags, see Section 5.2 of [RFC5444].

MAL: Message Address Length, see Section 5.2 of [RFC5444].

MTLVF: Message TLV Flags, see Section 5.4.1 of [RFC5444].

MTLVExt: Message TLV Type Extension, see Section 5.4.1 of [RFC5444].

AF : $\quad$ Address Block Flags, see Section 5.3 of [RFC5444].

ABTLV: Address Block TLV, see Section 5.4 of [RFC5444].

ABTLVF: Address Block TLV Flags, see section 5.4.1 of [RFC5444].

Example Message with ICV - Legend

13. IANA Considerations

The IANA registrations for TLV Types and the TLV type extension registries given in this specification replace the identical registrations and registries from [RFC6622].

This specification defines the following TLV Types, replacing the original specifications in [RFC6622]:

- Two Packet TLV Types, which have been allocated from the 0-223 range of the "Packet TLV Types" repository of [RFC5444], as specified in Table 1 .

- Two Message TLV Types, which have been allocated from the 0-127 range of the "Message TLV Types" repository of [RFC5444], as specified in Table 2 .

- Two Address Block TLV Types, which have been allocated from the 0-127 range of the "Address Block TLV Types" repository of [RFC5444], as specified in Table 3.

This specification updates the following registries that were created in [RFC6622]:

- A type extension registry for each of these TLV types with values as listed in Tables 1,2 , and 3 .

The following terms are used as defined in [BCP26]: "Namespace", "Registration", and "Designated Expert".

The following policy is used as defined in [BCP26]: "Expert Review".

13.1. Expert Review: Evaluation Guidelines

For TLV type extensions registries where an Expert Review is required, the Designated Expert SHOULD take the same general recommendations into consideration as those specified by [RFC5444]. 
For both TIMESTAMP and ICV TLVs, functionally similar extensions for Packet, Message, and Address Block TLVs SHOULD be numbered identically.

\subsection{Packet TLV Types}

IANA has, in accordance with [RFC6622], made allocations from the "Packet TLV Types" namespace of [RFC5444] for the Packet TLVs specified in Table 1. IANA has modified this allocation as indicated.

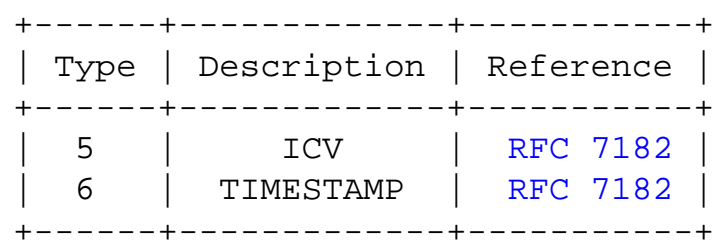

Table 1: Packet TLV Types

13.3. Message TLV Types

IANA has, in accordance with [RFC6622], made allocations from the "Message TLV Types" namespace of [RFC5444] for the Message TLVs specified in Table 2. IANA has modified this allocation as indicated.

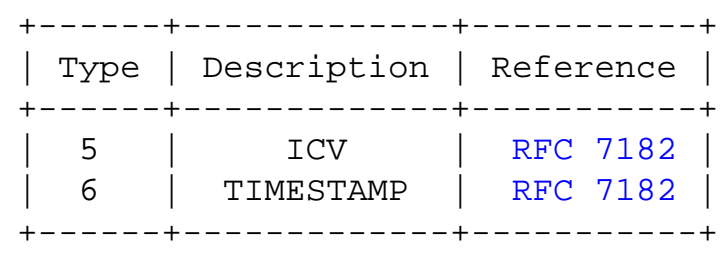

Table 2: Message TLV Types

\subsection{Address Block TLV Types}

IANA has, in accordance with [RFC6622], made allocations from the "Address Block TLV Types" namespace of [RFC5444] for the Packet TLVs specified in Table 3 . IANA has modified this allocation as indicated. 


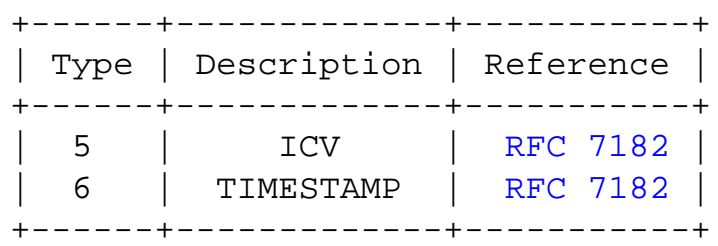

Table 3: Address Block TLV Types

\subsection{ICV Packet TLV Type Extensions}

IANA has, in accordance with [RFC6622], made allocations from the "ICV Packet TLV Type Extensions" namespace of [RFC6622] for the Packet TLVs specified in Table 4. IANA has modified this allocation (including defining type extension $=2$ ) as indicated.

\begin{tabular}{|c|c|c|}
\hline $\begin{array}{c}\text { Type } \\
\text { Extension }\end{array}$ & Description & Reference \\
\hline 0 & ICV of a packet & RFC 7182 \\
\hline 1 & $\begin{array}{c}\text { ICV, using a cryptographic function and a } \\
\text { hash function, as specified in section } 12 \\
\text { of this document }\end{array}$ & RFC 7182 \\
\hline $3-251$ & $\begin{array}{l}\text { ICV, using a cryptographic function and a } \\
\text { hash function, and including the IP } \\
\text { datagram source address, as specified in } \\
\text { Section } 12 \text { of this document } \\
\text { Unassigned; Expert Review }\end{array}$ & RFC 7182 \\
\hline $252-255$ & Reserved for Experimental Use & RFC 7182 \\
\hline
\end{tabular}

Table 4: ICV Packet TLV Type Extensions

More than one ICV Packet TLV with the same type extension MAY be included in a packet if these represent different ICV calculations (e.g., with type extension 1 or 2 and different cryptographic function and/or hash function or with a different key identifier). ICV Packet TLVs that carry what is declared to be the same information MUST NOT be included in the same packet.

\subsection{TIMESTAMP Packet TLV Type Extensions}

IANA has, in accordance with [RFC6622], made allocations from the "TIMESTAMP Packet TLV Type Extensions" namespace of [RFC6622] for the Packet TLVs specified in Table 5. IANA has modified this allocation as indicated. 


\begin{tabular}{|c|c|c|}
\hline $\begin{array}{l}\text { Type } \\
\text { Extension }\end{array}$ & Description & Reference \\
\hline 0 & $\begin{array}{l}\text { Unsigned timestamp of arbitrary length, } \\
\text { given by the TLV Length field. The MANET } \\
\text { routing protocol has to define how to } \\
\text { interpret this timestamp }\end{array}$ & RFC 7182 \\
\hline 1 & $\begin{array}{c}\text { Unsigned } 32 \text {-bit timestamp, as specified } \\
\text { in [IEEE1003.1-2008] }\end{array}$ & RFC 7182 \\
\hline 2 & $\begin{array}{c}\text { NTP timestamp format, as specified in } \\
\text { [RFC5905] }\end{array}$ & RFC 7182 \\
\hline 3 & $\begin{array}{c}\text { Signed timestamp of arbitrary length with } \\
\text { no constraints such as monotonicity. In } \\
\text { particular, it may represent any random } \\
\text { value }\end{array}$ & RFC 7182 \\
\hline $4-251$ & Unassigned; Expert Review & \\
\hline $252-255$ & Reserved for Experimental Use & RFC 7182 \\
\hline
\end{tabular}

Table 5: TIMESTAMP Packet TLV Type Extensions

More than one TIMESTAMP Packet TLV with the same type extension MUST NOT be included in a packet.

\subsection{ICV Message TLV Type Extensions}

IANA has, in accordance with [RFC6622], made allocations from the "ICV Message TLV Type Extensions" namespace of [RFC6622] for the Message TLVs specified in Table 6 . IANA has modified this allocation (including defining type extension $=2$ ) as indicated. 


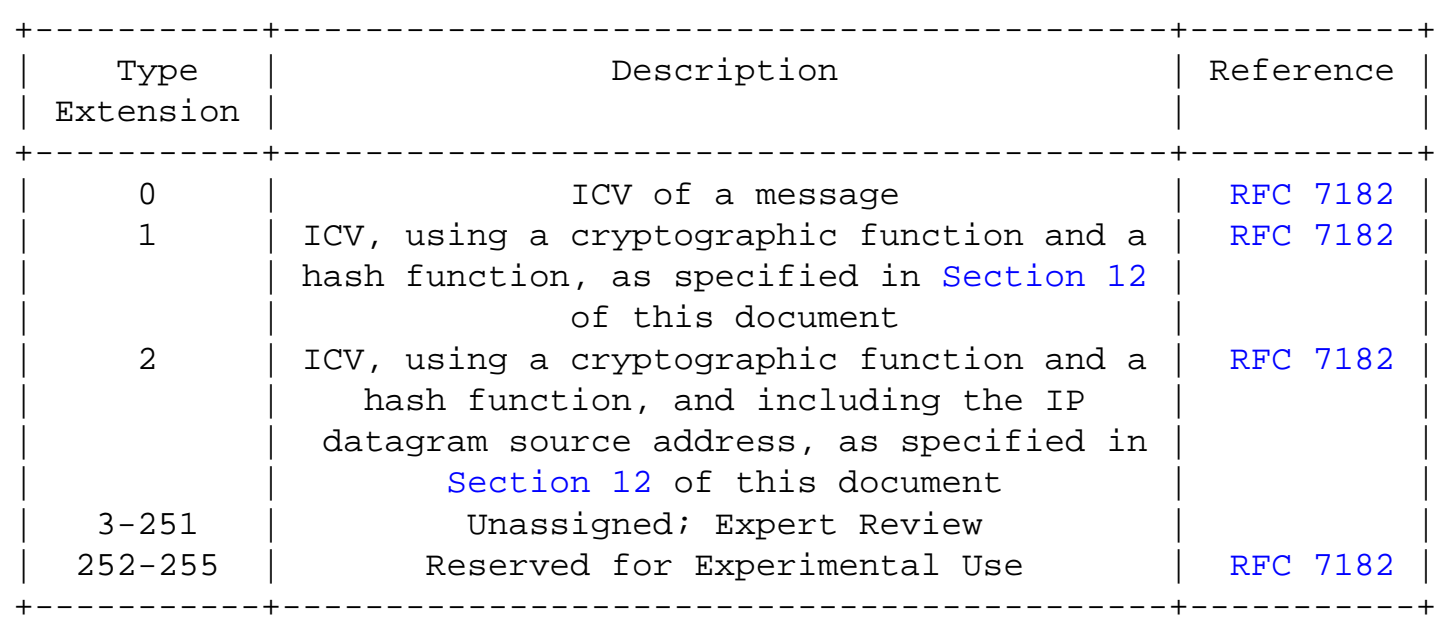

Table 6: ICV Message TLV Type Extensions

More than one ICV Message TLV with the same type extension MAY be included in a message if these represent different ICV calculations (e.g., with type extension 1 or 2 and different cryptographic function and/or hash function or with a different key identifier). ICV Message TLVs that carry what is declared to be the same information MUST NOT be included in the same message.

\subsection{TIMESTAMP Message TLV Type Extensions}

IANA has, in accordance with [RFC6622], made allocations from the "TIMESTAMP Message TLV Type Extensions" namespace of [RFC6622] for the Message TLVs specified in Table 7. IANA has modified this allocation as indicated. 


\begin{tabular}{|c|c|c|}
\hline $\begin{array}{l}\text { Type } \\
\text { Extension }\end{array}$ & Description & Reference \\
\hline 0 & $\begin{array}{l}\text { Unsigned timestamp of arbitrary length, } \\
\text { given by the TLV Length field. The MANET } \\
\text { routing protocol has to define how to } \\
\text { interpret this timestamp }\end{array}$ & RFC 7182 \\
\hline 1 & $\begin{array}{c}\text { Unsigned 32-bit timestamp, as specified } \\
\text { in POSIX [IEEE1003.1-2008] }\end{array}$ & RFC 7182 \\
\hline 2 & $\begin{array}{c}\text { NTP timestamp format, as specified in } \\
\text { [RFC5905] }\end{array}$ & RFC 7182 \\
\hline 3 & $\begin{array}{c}\text { Signed timestamp of arbitrary length with } \\
\text { no constraints such as monotonicity. In } \\
\text { particular, it may represent any random } \\
\text { value }\end{array}$ & RFC 7182 \\
\hline $4-251$ & Unassigned; Expert Review & \\
\hline $252-255$ & Reserved for Experimental Use & RFC 7182 \\
\hline
\end{tabular}

Table 7: TIMESTAMP Message TLV Type Extensions

More than one TIMESTAMP Message TLV with the same type extension MUST NOT be included in a message.

\subsection{ICV Address Block TLV Type Extensions}

IANA has, in accordance with [RFC6622], made allocations from the "ICV Address Block TLV Type Extensions" namespace of [RFC6622] for the Address Block TLVs specified in Table 8. IANA has modified this allocation (including defining type extension $=2$ ) as indicated. 


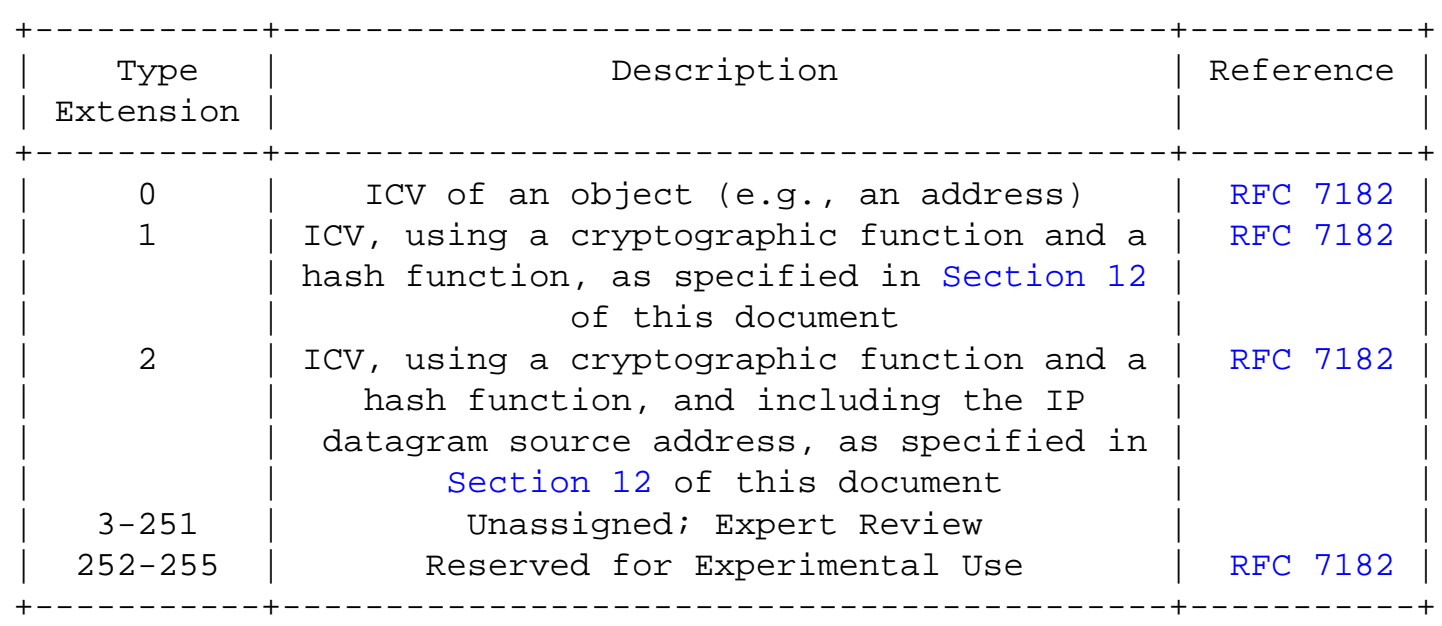

Table 8: ICV Address Block TLV Type Extensions

More than one ICV Address Block TLV with the same type extension MAY be associated with an address if these represent different ICV calculations (e.g., with type extension $=1$ or type extension $=2$ and different cryptographic function and/or hash function or with a different key identifier). ICV Address Block TLVs that carry what is declared to be the same information MUST NOT be associated with the same address.

\subsection{TIMESTAMP Address Block TLV Type Extensions}

IANA has, in accordance with [RFC6622], made allocations from the "TIMESTAMP Address Block TLV Type Extensions" namespace of [RFC6622] for the Address Block TLVs specified in Table 9. IANA has modified this allocation as indicated. 


\begin{tabular}{|c|c|c|}
\hline $\begin{array}{l}\text { Type } \\
\text { Extension }\end{array}$ & Description & Reference \\
\hline 0 & $\begin{array}{l}\text { Unsigned timestamp of arbitrary length, } \\
\text { given by the TLV Length field. The MANET } \\
\text { routing protocol has to define how to } \\
\text { interpret this timestamp }\end{array}$ & RFC 7182 \\
\hline 1 & $\begin{array}{c}\text { Unsigned 32-bit timestamp, as specified } \\
\text { in POSIX [IEEE1003.1-2008] }\end{array}$ & RFC 7182 \\
\hline 2 & $\begin{array}{c}\text { NTP timestamp format, as specified in } \\
\text { [RFC5905] }\end{array}$ & RFC 7182 \\
\hline 3 & $\begin{array}{c}\text { Signed timestamp of arbitrary length with } \\
\text { no constraints such as monotonicity. In } \\
\text { particular, it may represent any random } \\
\text { value }\end{array}$ & RFC 7182 \\
\hline $4-251$ & Unassigned; Expert Review & \\
\hline $252-255$ & Reserved for Experimental Use & RFC 7182 \\
\hline
\end{tabular}

Table 9: TIMESTAMP Address Block TLV Type Extensions

More than one TIMESTAMP Address Block TLV with the same type extension MUST NOT be associated with any address.

\subsection{Hash Functions}

IANA has, in accordance with [RFC6622], created a registry for hash functions that can be used when creating an ICV, as specified in Section 12 of this document. The initial assignments and allocation policies are specified in Table 10. IANA has modified this allocation as indicated. 


\begin{tabular}{|c|c|c|c|}
\hline Value & Algorithm & Description & Reference \\
\hline 0 & none & $\begin{array}{c}\text { The "identity function": The } \\
\text { hash value of an object is the } \\
\text { object itself }\end{array}$ & RFC 7182 \\
\hline 1 & SHA-1 & [NIST-FIPS-180-4] & RFC 7182 \\
\hline 2 & SHA -224 & [NIST-FIPS-180-4] & RFC 7182 \\
\hline 3 & SHA -256 & [NIST-FIPS-180-4] & RFC 7182 \\
\hline 4 & $\mathrm{SHA}-384$ & [NIST-FIPS-180-4] & RFC 7182 \\
\hline 5 & SHA -512 & [NIST-FIPS-180-4] & RFC 7182 \\
\hline $6-251$ & & Unassigned; Expert Review & \\
\hline $252-255$ & & Reserved for Experimental Use & RFC 7182 \\
\hline
\end{tabular}

Table 10: Hash Function Registry

\subsection{Cryptographic Functions}

IANA has, in accordance with [RFC6622], created a registry for the cryptographic functions, as specified in section 12 of this document. Initial assignments and allocation policies are specified in Table 11. IANA has modified this allocation as indicated.

\begin{tabular}{|c|c|c|c|}
\hline Value & Algorithm & Description & Reference \\
\hline 0 & none & $\begin{array}{l}\text { The "identity function": The } \\
\text { value of an encrypted hash is } \\
\text { the hash itself }\end{array}$ & RFC 7182 \\
\hline 1 & RSA & {$[\mathrm{RFC} 3447]$} & RFC 7182 \\
\hline 2 & $\mathrm{DSA}$ & [NIST-FIPS-186-4] & RFC 7182 \\
\hline 3 & HMAC & {$[\mathrm{RFC} 2104]$} & RFC 7182 \\
\hline 4 & 3DES & {$[N I S T-S P-800-67]$} & RFC 7182 \\
\hline 5 & $\mathrm{AES}$ & [NIST-FIPS-197] & RFC 7182 \\
\hline 6 & ECDSA & {$[\mathrm{RFC} 6090]$} & RFC 7182 \\
\hline $7-251$ & & Unassigned; Expert Review & \\
\hline $252-255$ & & Reserved for Experimental Use & RFC 7182 \\
\hline
\end{tabular}

Table 11: Cryptographic Function Registry 
14. Security Considerations

This document does not specify a protocol. It provides a syntactical component for cryptographic ICVs of messages and packets, as defined in [RFC5444]. It can be used to address security issues of a MANET routing protocol or MANET routing protocol extension. As such, it has the same security considerations as [RFC5444].

In addition, a MANET routing protocol or MANET routing protocol extension that uses this specification MUST specify how to use the framework and the TLVs presented in this document. In addition, the protection that the MANET routing protocol or MANET routing protocol extensions attain by using this framework MUST be described.

As an example, a MANET routing protocol that uses this component to reject "badly formed" or "insecure" messages if a control message does not contain a valid ICV SHOULD indicate the security assumption that if the ICV is valid, the message is considered valid. It also SHOULD indicate the security issues that are counteracted by this measure (e.g., link or identity spoofing) as well as the issues that are not counteracted (e.g., compromised keys).

\section{Acknowledgements}

The authors would like to thank Bo Berry (Cisco), Alan Cullen (BAE Systems), Justin Dean (NRL), Paul Lambert (Marvell), Jerome Milan (Ecole Polytechnique), and Henning Rogge (FGAN) for their constructive comments on [RFC6622].

The authors also appreciate the detailed reviews of [RFC6622] from the Area Directors, in particular Stewart Bryant (Cisco), Stephen Farrell (Trinity College Dublin), and Robert Sparks (Tekelec), as well as Donald Eastlake (Huawei) from the Security Directorate.

The authors would like to thank Justin Dean (NRL) and Henning Rogge (FGAN) for their constructive comments on this specification. 
16. References

16.1. Normative References

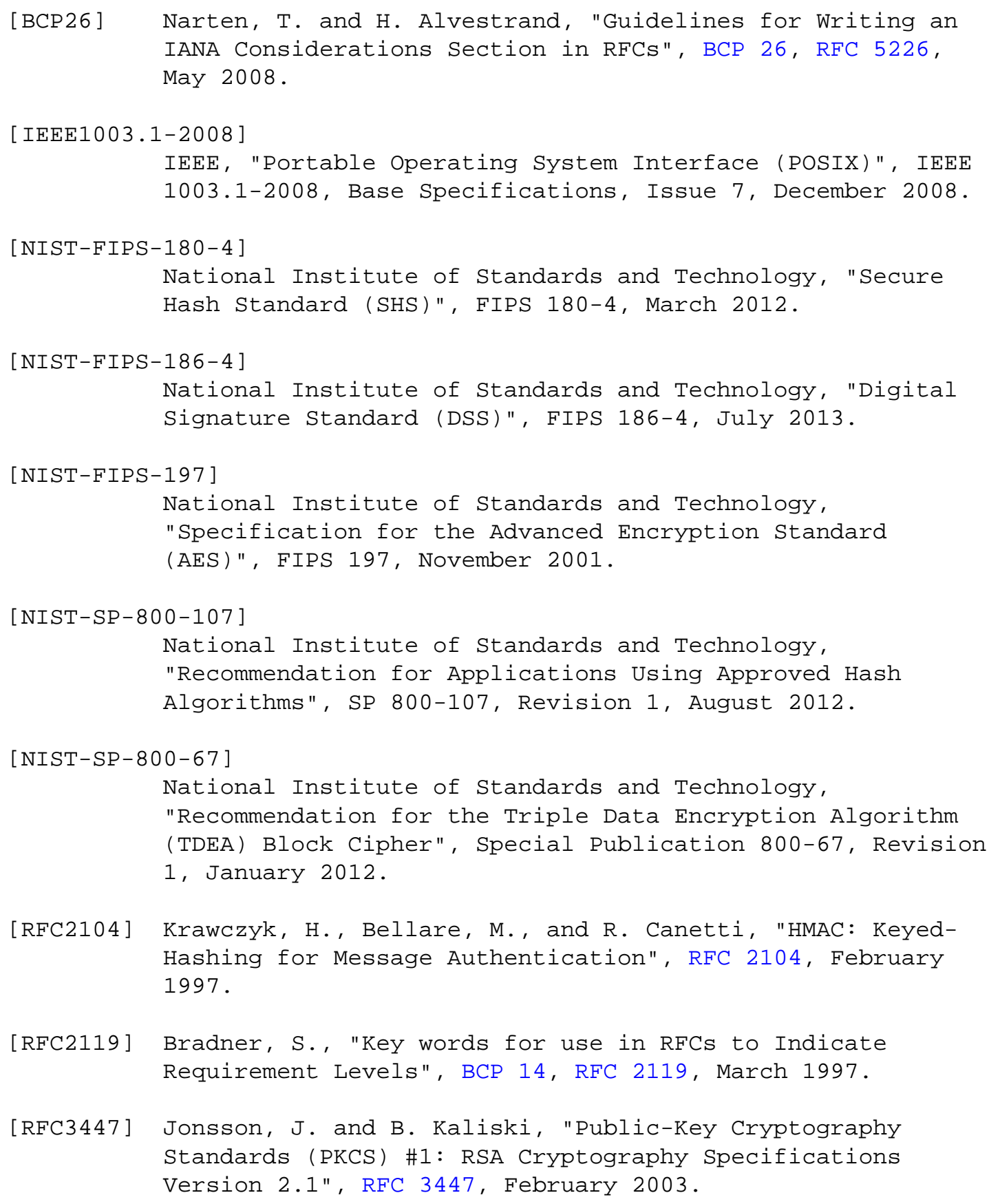

[RFC2104] Krawczyk, H., Bellare, M., and R. Canetti, "HMAC: KeyedHashing for Message Authentication", RFC 2104, February 1997.

[RFC2119] Bradner, S., "Key words for use in RFCs to Indicate Requirement Levels", BCP 14, RFC 2119, March 1997.

[RFC3447] Jonsson, J. and B. Kaliski, "Public-Key Cryptography Standards (PKCS) \#1: RSA Cryptography Specifications Version 2.1", RFC 3447, February 2003. 
[RFC4493] Song, JH., Poovendran, R., Lee, J., and T. Iwata, "The AES-CMAC Algorithm", RFC 4493, June 2006.

[RFC5444] Clausen, T., Dearlove, C., Dean, J., and C. Adjih, "Generalized Mobile Ad Hoc Network (MANET) Packet/Message Format", RFC 5444, February 2009.

[RFC5905] Mills, D., Martin, J., Burbank, J., and W. Kasch, "Network Time Protocol Version 4: Protocol and Algorithms Specification", RFC 5905, June 2010.

[RFC6090] McGrew, D., Igoe, K., and M. Salter, "Fundamental Elliptic Curve Cryptography Algorithms", RFC 6090, February 2011.

16.2. Informative References

[RFC6130] Clausen, T., Dearlove, C., and J. Dean, "Mobile Ad Hoc Network (MANET) Neighborhood Discovery Protocol (NHDP)", RFC 6130, April 2011.

[RFC6622] Herberg, U. and T. Clausen, "Integrity Check Value and Timestamp TLV Definitions for Mobile Ad Hoc Networks (MANETS)", RFC 6622, May 2012.

[RFC7181] Clausen, T., Dearlove, C., Jacquet, P., and U. Herberg, "The Optimized Link state Routing Protocol Version 2", RFC 7181, April 2014. 


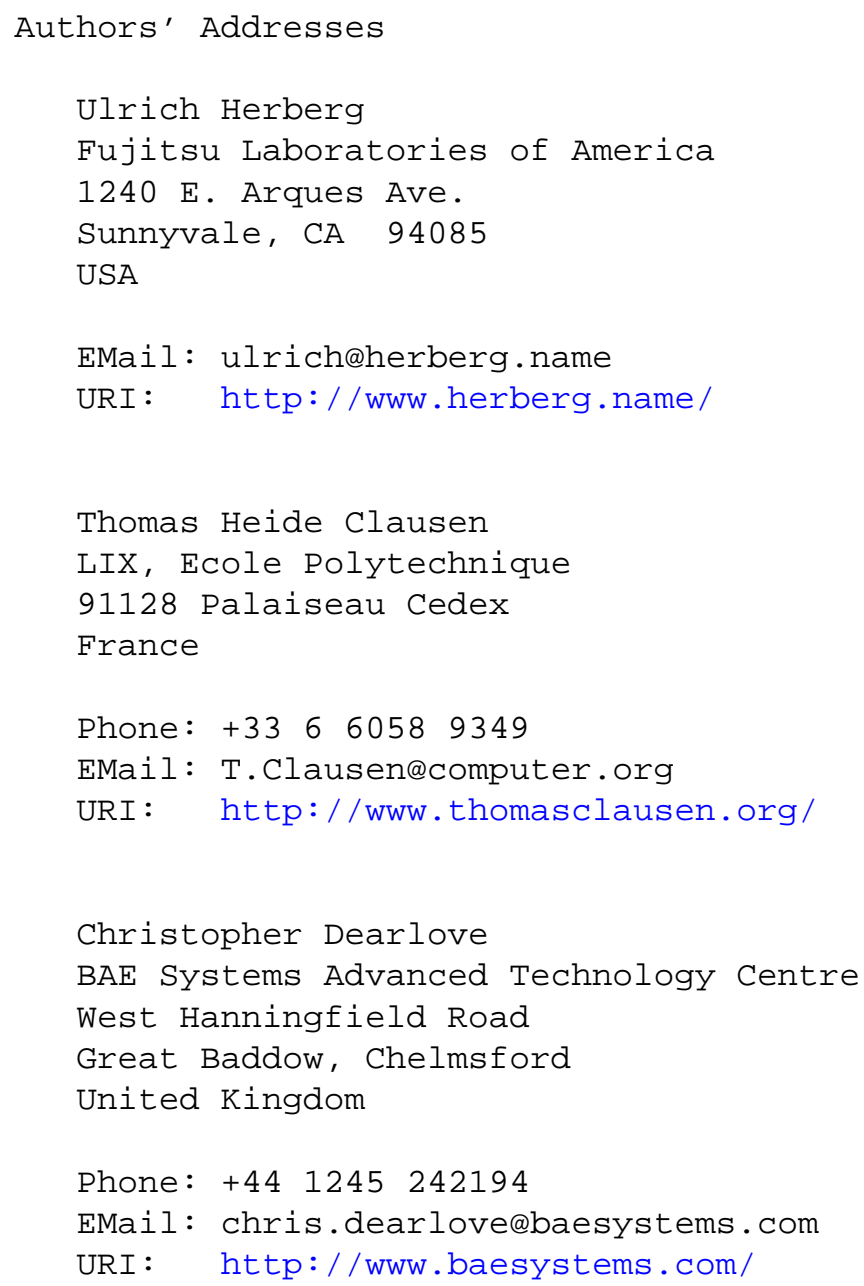

